\title{
Targeted ablation of IKK2 improves skeletal muscle strength, maintains mass, and promotes regeneration
}

\author{
Foteini Mourkioti, ${ }^{1}$ Paschalis Kratsios, ${ }^{1}$ Tom Luedde, ${ }^{1,2}$ Yao-Hua Song, ${ }^{3}$ Patrick Delafontaine, ${ }^{3}$ \\ Raffaella Adami, ${ }^{4}$ Valeria Parente, ${ }^{4}$ Roberto Bottinelli, ${ }^{4}$ Manolis Pasparakis, ${ }^{1,2}$ and Nadia Rosenthal ${ }^{1}$ \\ ${ }^{1}$ European Molecular Biology Laboratory (EMBL) Mouse Biology Unit, Campus “A. Buzzati-Traverso," Monterotondo-Scalo, Italy. \\ 2Department of Mouse Genetics and Inflammation, Institute for Genetics, University of Cologne, Cologne, Germany. \\ ${ }^{3}$ Section of Cardiology, Tulane University School of Medicine, New Orleans, Louisiana, USA. \\ ${ }^{4}$ Department of Experimental Medicine, Human Physiology Unit, University of Pavia, Pavia, Italy.
}

\begin{abstract}
$\mathrm{NF}-\kappa \mathrm{B}$ is a major pleiotropic transcription factor modulating immune, inflammatory, cell survival, and proliferative responses, yet the relevance of NF- $\kappa B$ signaling in muscle physiology and disease is less well documented. Here we show that muscle-restricted NF- $\kappa B$ inhibition in mice, through targeted deletion of the activating kinase inhibitor of NF- $\kappa \mathrm{B}$ kinase 2 (IKK2), shifted muscle fiber distribution and improved muscle force. In response to denervation, IKK2 depletion protected against atrophy, maintaining fiber type, size, and strength, increasing protein synthesis, and decreasing protein degradation. IKK2-depleted mice with a muscle-specific transgene expressing a local Igf-1 isoform (mIgf-1) showed enhanced protection against muscle atrophy. In response to muscle damage, IKK2 depletion facilitated skeletal muscle regeneration through enhanced satellite cell activation and reduced fibrosis. Our results establish IKK2/NF- $\kappa$ B signaling as an important modulator of muscle homeostasis and suggest a combined role for IKK inhibitors and growth factors in the therapy of muscle diseases.
\end{abstract}

\section{Introduction}

Tissue remodeling is an important physiological process that allows skeletal muscle to respond to environmental demands, promoting adaptive changes in cytoarchitecture and protein composition in response to a variety of stimuli. A host of extracellular agonists, receptors, protein kinases, intermediate molecules, and transcription factors participate in signal transduction pathways, promoting specific cellular responses to environmental cues. Perturbations in these pathways can result in chronic protein degradation, one of the most devastating consequences of defects in muscle survival mechanisms.

Disruption of skeletal muscle homeostasis can appear spontaneously at all ages, resulting in weakness, muscle atrophy, and degeneration. The genetic defects responsible for muscle dysfunction in several inherited pathologies have been well characterized, as in muscular dystrophies where the disease results from mutations in the giant gene encoding dystrophin, a structural protein (1). However, muscle wasting is often a secondary consequence of other pathological states. For example, cardiac cachexia leads to progressive muscle atrophy, which has been increasingly appreciated as a powerful negative predictive factor in heart failure $(2,3)$. Moreover, most cancer patients suffer from muscle degeneration (4), which also occurs in patients with dysfunction of the immune system (5).

Nonstandard abbreviations used: CSA, cross-sectional area; CTX, cardiotoxin; EDL, extensor digitorum longus; GSK3 $\alpha / \beta$, glycogen synthase kinase $3 \alpha / \beta$; I $\mathrm{B}$, inhibitor

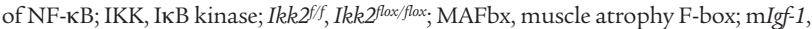
muscle-specific transgene expressing a local Igf-1 isoform; mko, muscle knockout; mTOR, mammalian target of rapamycin; MuRF1, muscle RING finger 1; NEMO, NF-אB essential modulator; nLacZ, nuclear LacZ; p, phosphorylated; Q, quadriceps; TA, tibialis anterior.

Conflict of interest: The authors have declared that no conflict of interest exists. Citation for this article: J. Clin. Invest. 116:2945-2954 (2006). doi:10.1172/JCI28721.
Despite its widespread occurrence, the molecular underpinnings of muscle atrophy have remained elusive. Subtle alterations in signaling pathways have been identified as leading to significant defects in muscle metabolism, yet the field has been stalled in devising successful therapeutic strategies for treatment of this debilitating and often fatal group of human ailments. The complexity of muscle types, the intimate relationship between structural integrity and mechanical function, and the sensitivity of skeletal muscle to metabolic perturbations have impeded rapid progress in successful clinical intervention. The increasingly limited regenerative properties of aging skeletal muscle further compound the devastating effects of wasting.

Pathological loss of muscle mass occurs primarily through enhanced protein breakdown mediated by ubiquitin, a small, highly conserved protein that becomes covalently linked to lysine residues of intracellular proteins destined for degradation (6). The critical regulators of protein ubiquitination are tissue-specific E3 ligases $(7,8)$ that catalyze the transfer of activated ubiquitin, selectively targeting specific proteins for rapid degradation through the $26 \mathrm{~S}$ proteasome (6). The crucial role of the ubiquitin-proteasome pathway has been demonstrated in several models of muscular atrophy that include starvation (7), uremia (9), diabetes (10), and sepsis (11). The characterization of molecular pathways that regulate muscle catabolism through control of ubiquitin-proteasome-mediated degradation is of critical importance in designing candidate gene targets for the attenuation of muscle wasting.

Maintenance of muscle mass is also profoundly influenced by secondary factors, such as inflammation. Indeed, systemic inflammation rather than disuse has been cited as the primary cause of muscle wasting during aging and chronic disorders (12). Among the pathways controlling inflammation, those activating transcription factor NF- $\kappa \mathrm{B}$ play a major pleiotropic role in the modulation 
A

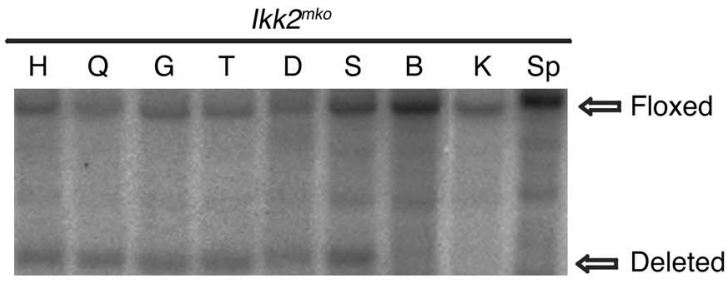

B

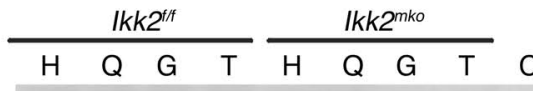

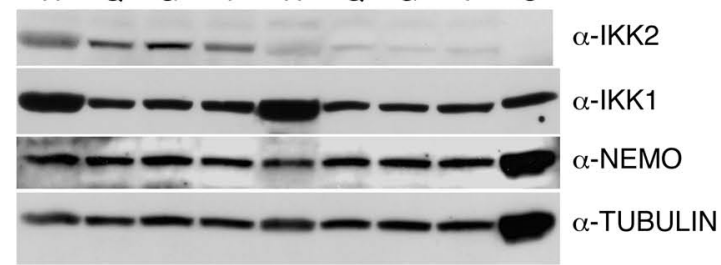

E
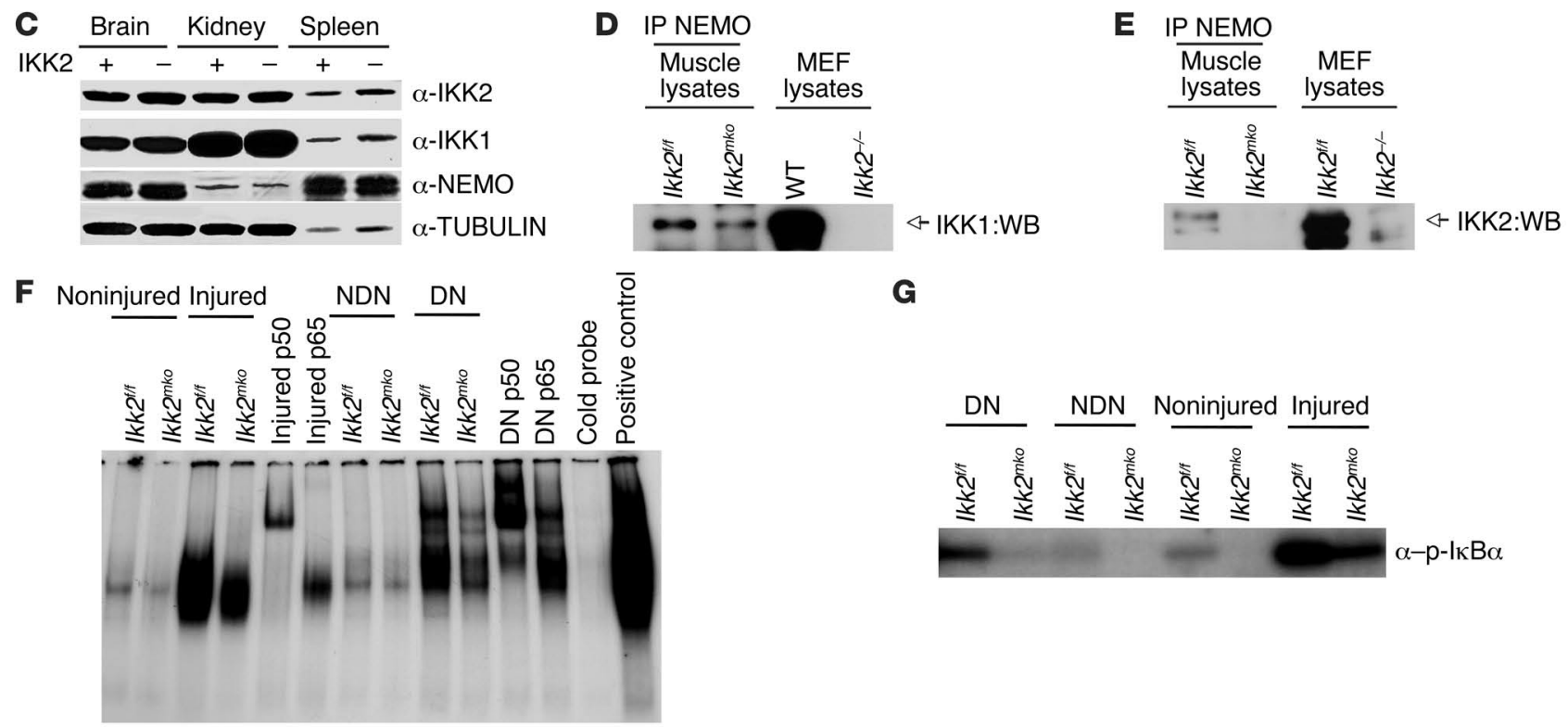

G

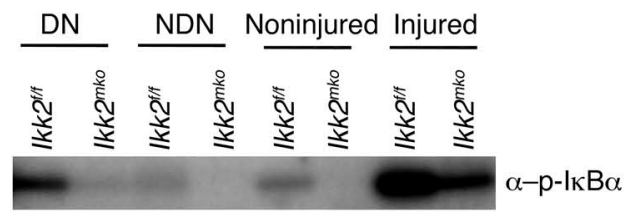

Figure 1

Comparison of NF-kB activation in $I k k 2^{f / f}$ and $l k k 2^{m k o}$ muscles. (A) Deletion of the floxed alleles only in $l k k 2^{m k o}$ skeletal muscles as shown by Southern blot analysis on genomic DNA isolated from different tissues. (B) Decreased expression of IKK2 in different lkk2mko muscles, shown by immunoblotting of total muscle extracts. The other subunits, IKK1 and NEMO, remain intact. (C) Unchanged levels of IKK proteins in other tissues. H, heart; G, gastrocnemius; T, TA; D, diaphragm; S, soleus; B, brain; K, kidney; Sp, spleen; C, control from brain-specific IKK2 deletion. (D) IP in $300 \mu \mathrm{g}$ of whole-muscle extracts from $I k 2^{t / f}$ and $I k k 2^{m k o}$ mice was performed with a polyclonal antibody against NEMO, followed by Western blot (WB) analysis with a monoclonal antibody against IKK1 or IKK2 as indicated. MEF, mouse embryonic fibroblasts. (E) Nuclear translocation of NF-KB was demonstrated by EMSA. (F) Evaluation of IKK activity. Total protein (300 $\mu \mathrm{g})$ from normal or challenged (injured or denervated) muscles was IP with a NEMO antibody and subjected to a kinase assay using a truncated glutathione S-transferase-IкBa (aa residues 1-54) protein as substrate. DN, denervated; NDN, nondenervated.

of immune, inflammatory, cell survival, and proliferative responses (13). In unstimulated cells, NF- $\mathrm{KB}$ is kept inactive via association with inhibitor of NF- $\kappa \mathrm{B}(\mathrm{I} \kappa \mathrm{B})$ proteins. Induced phosphorylation of

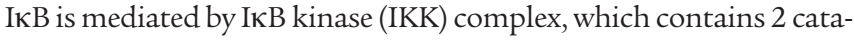
lytic subunits (IKK1/ $\alpha$ and IKK2/ $\beta$ ) (14) and a regulatory subunit termed NF-KB essential modulator/IKK $\gamma$ (NEMO/IKK $\gamma$ ) (15). Upon encountering a variety of stimuli, including TNF- $\alpha$, IL-1, and other growth factors, I $\mathrm{I} B \alpha$ is phosphorylated and degraded through the ubiquitination pathway, rendering NF- $\kappa \mathrm{B}$ free to accumulate in the nucleus and bind to its target genes (16). In muscle tissue cultures, $\mathrm{NF}-\kappa \mathrm{B}$ influences myogenic growth and differentiation $(17,18)$, downregulates MyoD expression $(19,20)$, and induces muscle atrophy in mice (21). However, less is known about the in vivo contribution of NF- $\mathrm{KB}$ to skeletal muscle development and regeneration.

To address the specific physiological role of proinflammatory signaling in skeletal muscle homeostasis and to bypass the embryonic lethality caused by systemic NF-אB depletion $(22,23)$, we targeted the IKK2 subunit of the IKK complex, using the Cre/ loxP recombination system, to restrict inactivation of the gene to muscle cells. We also took advantage of recent studies on the role of IGF-1 in the promotion of muscle regeneration and resolution of the inflammatory response (24). Here we report that mouse muscles depleted of NF- $\mathrm{BB}$ signaling exhibit an increase in muscle strength, maintain normal muscle physiology, block protein degradation under atrophy conditions, and display enhanced muscle regeneration in response to injury. Combined with supplemental IGF-1 in the form of a muscle-specific transgene expressing a local Igf-1 isoform (mIgf-1) (25), abrogation of NF- $\mathrm{KB}$ signaling provides an even greater protection against muscle atrophy. These results place the control of inflammatory pathways at center stage for clinical intervention in muscle atrophy and degeneration and underscore the potential power of combination therapies to counter the devastating consequences of muscle wasting.

\section{Results}

Characterization of Ikk2-depleted mice. We deleted the IKK2 subunit of the IKK complex by crossing Ikk2 flox/flox $\left(I k k 2^{f / f}\right)$ (26) mice expressing Cre recombinase under the control of the muscle creatine kinase 


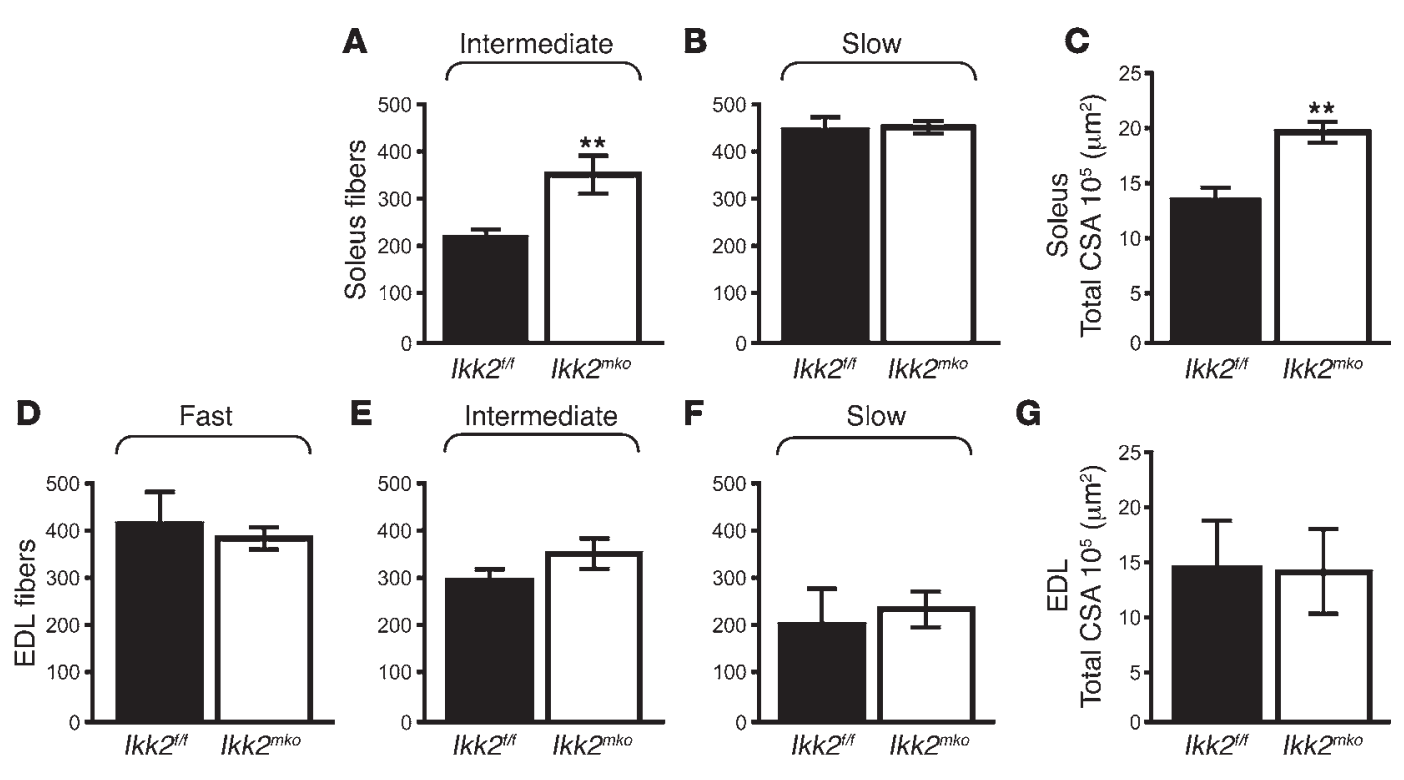

Figure 2

IKK2 muscle depletion increases number of intermediate fibers. (A and B) Increased number of intermediate fibers in $/ k k 2^{m k o}$ soleus with similar number of slow fibers. (C) Mean percentage increase in $l k k 2^{m k o}$ soleus total CSA. (D-F) The number of intermediate fibers in the $I k k 2^{m k o}$ EDL was modestly but not significantly increased while the number of other fiber types remained unaltered. (G) Mean percentage of total CSA of the EDL was similar between control and $l k k 2^{m k o}$ animals. ${ }^{* *}$ Mean values differ significantly from those of control $(P<0.002)$.

(MCK) promoter (27). Ikk2 $2^{\text {muscle }}$ knockout $\left(I k k 2^{\text {mko }}\right.$ ) animals were viable and developed normally into adulthood. Southern blot analysis on genomic DNA from different tissues revealed $70 \%$ deletion of the floxed alleles specifically in the muscles of $I k k 2^{m k o}$ animals (Figure 1A). Western blot analysis of total muscle extracts showed significant reduction of the IKK2 protein in muscles of Ikk2 $2^{\text {mo }}$ animals while the levels of the other subunits in the IKK complex (IKK1 and NEMO) remained unchanged (Figure 1B). Moreover, expression of all IKK subunits in other tissues was not altered (Figure 1C), confirming that $I k k 2^{m k o}$ mice have reduced expression of the IKK2 subunit specifically in skeletal muscles. To further examine the IKK protein complex composition in Cre-negative control mice (Ikk2f/f) and $I k k 2^{m k o}$ mice, muscle protein extracts were immunoprecipitated with a NEMO antibody. As expected, no IKK2 was detectable in the IKK complex in Ikk2 $2^{m k o}$ mice whereas IKK1 protein could still be detected (Figure 1D).

To test whether IKK2 muscle depletion affected NF-KB activity in steady state or challenged conditions, we induced denervation by cutting the sciatic nerve, resulting in disuse of muscles in the lower leg (8). We also examined regenerating muscles by injecting cardiotoxin (CTX) in the tibialis anterior (TA) and the quadriceps (Q) (25). Nuclear protein extracts from steady state as well as challenged muscles (denervation or CTX injury) were analyzed using an EMSA. As shown in Figure 1E, moderate NF- $\mathrm{BB}$ activation in Ikk2f/f became pronounced in denervated or CTX-injured muscle while both stimuli failed to induce comparable levels of NF-кB DNA-binding activity in $I k k 2^{m k o}$ muscle. Finally, using a truncated IкB protein as substrate, we determined that kinase activity of the IKK complex was reduced in $I k k 2^{m k o}$ muscles in both challenges (Figure $1 \mathrm{~F}$ ), further underscoring the effect of NF- $\mathrm{KB}$ inactivation in Ikk2 $2^{\text {mko }}$ muscles.

Inbibition of IKK2 alters fiber number and improves muscle strength. To determine whether NF-кB inhibition affected the physiology of skeletal muscle, direct comparison of fiber number, type, and size was performed for the soleus (Figure 2, A and B) (contains 2 types of fiber: slow and intermediate) and the extensor digitorum longus (EDL) muscles (Figure 2, D-F) (contains all 3 types of fiber: fast, intermediate, and slow). The soleus in the Ikk2 $2^{\text {moo }}$ animals displayed a significant increase in the number of intermediate fibers (Figure 2A), with a concomitant increase in the total cross-sectional area (CSA) (Figure 2C). The number of intermediate fibers in the $I k k 2^{m k o}$ EDL was modestly, but not significantly, increased while the other fiber types as well as the total CSA remained unaltered (Figure 2, D-G). Moreover, the size of the individual fiber types in both muscles of the Ikk2 ${ }^{\text {mko }}$ animals remained unchanged (Supplemental Figure 1; supplemental material available online with this article; doi:10.1172/JCI28721DS1). Thus, IKK2 muscle depletion contributed to an increase in the number of intermediate fibers without affecting individual fiber size. Electrophysiological analysis of $I k k 2^{m k o}$ muscles revealed a corresponding increase in specific twitch and tetanic force (Figure 3, A and B). Moreover, in enforced treadmill tests, Ikk2 ${ }^{\text {mko }}$ mice could run longer distances (Figure 3C), providing further evidence that inhibition of IKK2 effectively increases the physical performance of the muscle.

Deletion of IKK2 subunit sustains muscle mass and strength and maintains fiber type during denervation-induced atropby. To explore the effect of IKK2 deletion on muscle loss, we examined atrophied muscles produced by sciatic nerve denervation. Control mice exhibited significant muscle atrophy at 7, 14, and 28 days after denervation (Figure 4, A and G) while at the same time points, the Ikk2 ${ }^{\text {mko }}$ muscle sustained more weight (Figure 4, B and G) in all leg muscles, with greatest differences observed in the soleus. To assess the role of IKK2

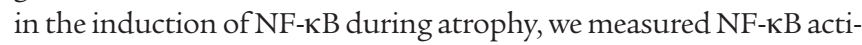
vation in the atrophied gastrocnemius 28 days after the operation. Control denervated muscles induced NF- $\mathrm{KB}$ activation while denervated Ikk2 ${ }^{\text {mko }}$ muscles showed impaired activation (EMSA, Figure 1E). Histological analysis was performed in the denervated muscles to 
A

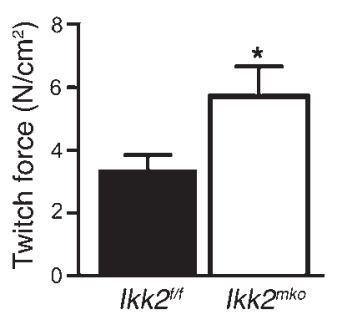

B

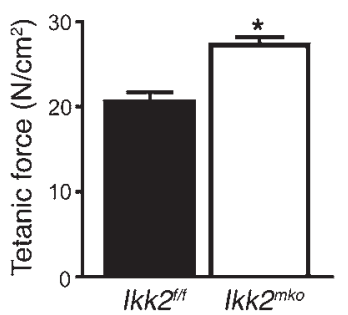

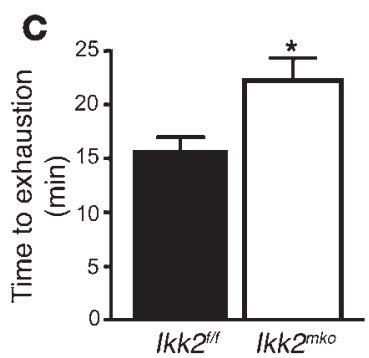

Figure 3

IKK2 muscle deletion improves muscle strength. (A) Increased specific twitch force of Ikk2 ${ }^{m k o}$ EDL in comparison with control EDL from littermates. (B) Lower maximum specific tetanic force of EDL in control $(n=15)$ versus $/ k 2^{m k o}$ animals $(n=11)$. (C) Time to exhaustion in treadmill test; control mice $(n=5)$ displayed a significantly lower performance than Ikk2 ${ }^{m k o}$ mice $(n=5)$. *Mean values differ significantly from those of control $(P<0.05)$.

determine whether the robust protective effect of IKK2 deletion on muscle mass (Figure 4, C-F) was due to maintenance of single fiber size. Indeed, in $I k k 2^{m k o}$ denervated muscle, fiber size was sustained compared with the corresponding control atrophied fibers, even as late as 28 days after denervation (Figure 5, A-C).

Atrophy due to denervation affected not only fiber size but also fiber type composition. To investigate the fiber type composition in $I k k 2^{f / f}$ and $I k k 2^{m k o}$ mice, NADH staining was performed on the soleus muscles of denervated versus sham-operated legs (Figure 5, $\mathrm{D}$ and E). Analysis of the I $\mathrm{k} k 2^{\mathrm{f} / \mathrm{f}}$ denervated muscle confirmed a decrease in slow fiber number and a parallel increase in intermediate-fast fiber number (Figure 5F) as reported for humans (28) and rats (29) while the number of slow and intermediate fibers was

not altered in the Ikk2 $2^{\text {mko }}$ denervated muscles (Figure 5F). Most importantly, Ikk2 ${ }^{m k o}$ atrophied muscles sustained their increased force (Figure $4 \mathrm{H}$ ), demonstrating protection from deterioration of muscle contractile properties under denervation conditions. Thus, NF-кB inhibition protects against atrophy by blocking muscle loss and preserving strength.

Deletion of IKK2 restabilizes muscle physiology under atrophy conditions. To determine which signaling pathways might be affected in NF- $\kappa \mathrm{B}-$ depleted muscles, phosphorylated protein levels in the atrophied muscles of control and $I k k 2^{\text {mko }}$ mice were systematically compared 28 days after denervation (Supplemental Figure 2). Protein synthetic pathways were generally downregulated in control muscles whereas in $I k k 2^{\text {mko }}$ denervated muscles, increased phosphorylation of Akt, glycogen synthase kinase $3 \alpha / \beta(\mathrm{GSK} 3 \alpha / \beta)$, mammalian target of rapamycin (mTOR), and p70S6K (Figure 6) was detected. Notably, denervated $I k k 2^{\text {mko }}$ muscles maintained decreased levels of muscle RING finger 1 (MuRF1), a critical regulator of muscle atrophy (8), while another regulator of protein degradation, atrogin-1/muscle atrophy F-box (atrogin-1/MAFbx) (30), was not affected (Figure 7, K and L). Together, these data indicate that IKK2 depletion stabilizes muscle homeostasis, both by promoting protein synthesis and attenuating protein degradation during muscle atrophy.

Double-mutant mIgf-1/Ikk2f/f animals show enhanced muscle atrophy protection. The effects of NF- $\mathrm{BB}$ inhibition on muscle atrophy are reminiscent of the action of IGF-1, which also protects against muscle atrophy through increased protein synthesis and
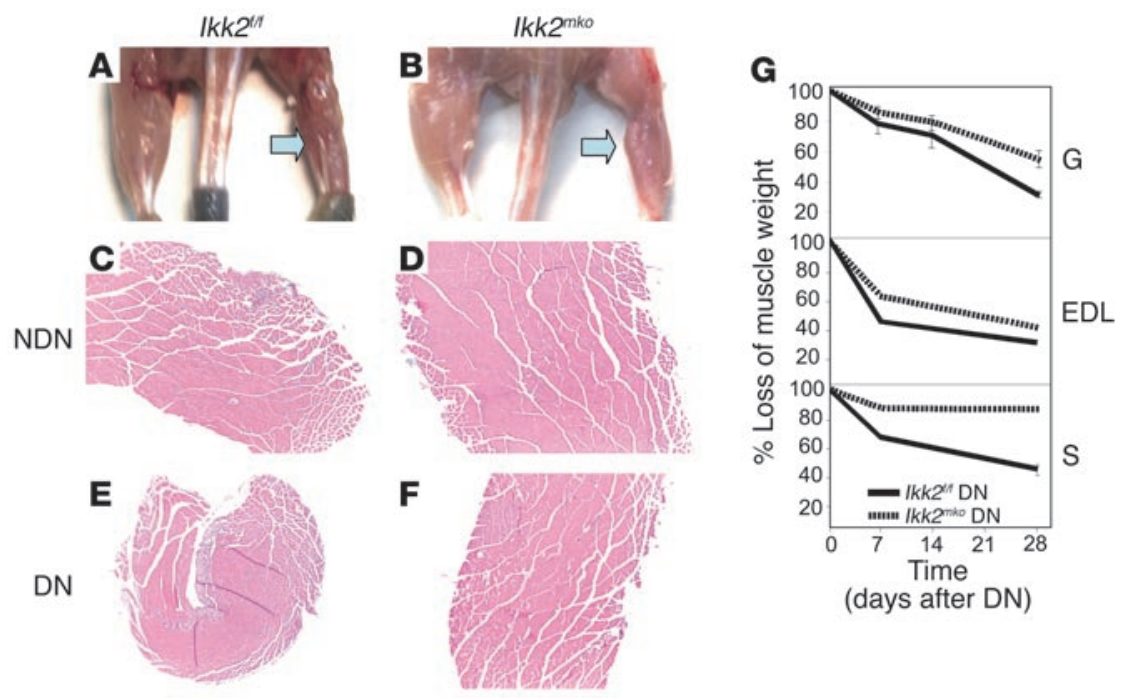

H

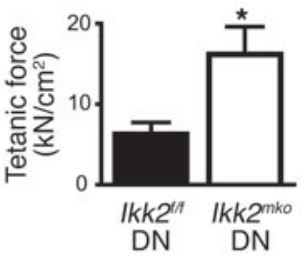

\section{Figure 4}

IKK2 muscle depletion maintains skeletal muscle mass and strength after denervation-induced atrophy. (A and B) Four-month-old mice 28 days after denervation procedure. Left arrow points to denervated leg (skin removed from the hind limbs). (C-F) Representative pictures are shown from cross sections taken from gastrocnemius (28 days after denervation) of both legs and stained with H\&E. Note the greater reduction of the muscle size of control compared with $I k 2^{m k o}$ atrophied gastrocnemius. (G) Quantitation of muscle mass in $l k k 2^{m k o}$ muscles (solid line) compared with those of control littermates (dashed line), at 7, 14, and 28 days after denervation. Note greater protection in $/ k k 2 m k o$ soleus. All values represent the weight of denervated muscle after normalization compared with the weight of sham-operated muscle $(n=5$ mice per time point) expressed as percentage. (H) Maximum EDL tetanic specific force of denervated control $(n=4)$ and denervated $l k k 2^{m k o}(n=4)$ muscle. ${ }^{*}$ Mean value differs significantly from that of control $(P<0.05)$. 

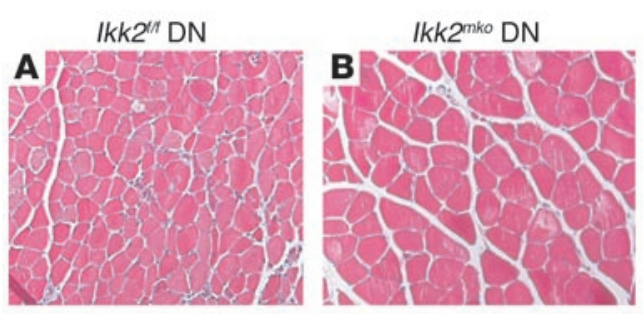

C
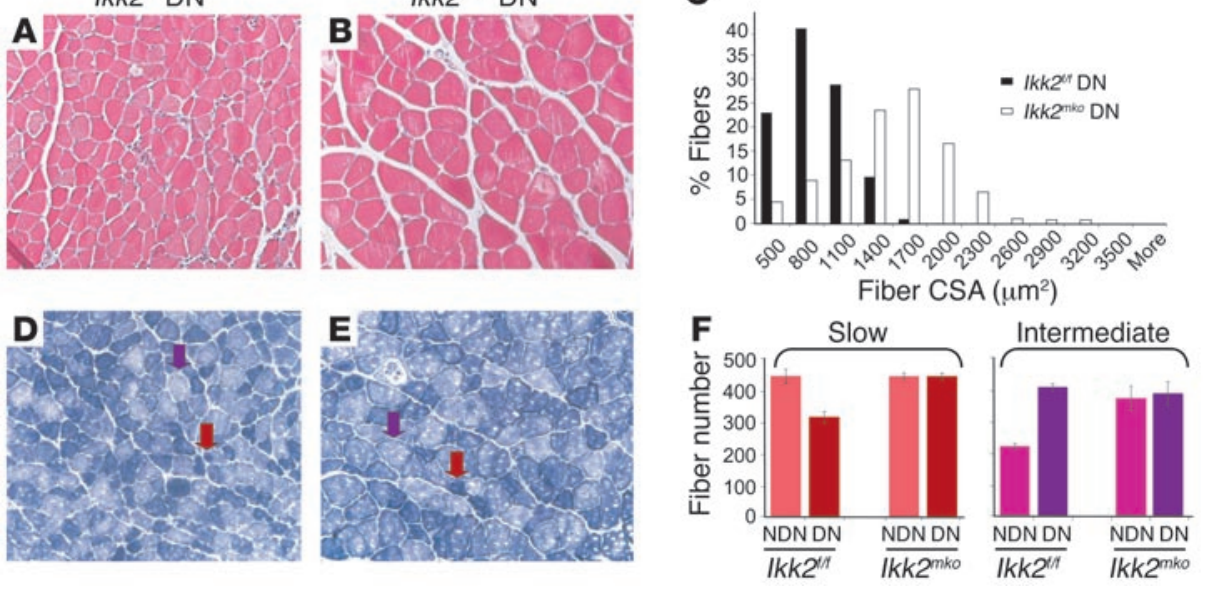

Figure 5

IKK2 muscle depletion protects fiber size and maintains fiber type. (A and B) Representative images of H\&E-stained atrophied muscle 28 days after denervation. Note the absence of angular atrophic fibers and the nuclear accumulation in $I k 2^{m k o}$ denervated muscles (C) Histogram (frequency distribution of fiber CSA) shows the maintenance of fiber size in Ikk2mko gastrocnemius (white bars) compared with the control atrophied muscles (black bars). ( $\mathbf{D}$ and $\mathbf{E}$ ) Representative images of $\mathrm{NADH}$ staining that mark slow fibers with dark blue (red arrows) and intermediate fibers with light blue (purple arrows). (F) Fiber-type distribution in denervated soleus muscles: $37.5 \%$ decrease in slow fibers in control muscles, which become intermediate. Note the maintenance of fiber-type distribution in $l k k 2^{m k o}$ denervated soleus.

decreased ubiquitination $(31,32)$. To investigate whether the combined effects of Ikk2 $2^{\text {mko }}$ depletion and IGF-1 supplementation are additive, genetic crosses were performed between the Ikk2 $2^{\text {moo }}$ mice and mice expressing a locally acting IGF-1 transgene in skeletal muscles (myosin light chain/mIgf-1) (25). Whereas mIgf-1 animals maintained muscle mass after denervation, the combined effects in $\mathrm{mIgf-1/Ikk2f/f}$ animals were even more pronounced, as shown by denervated gastrocnemius weight in a time course of 7,14 , and 28 days after the operation (Figure 7A). This enhanced protection was evident in the improved maintenance of muscle fiber size in comparison with either mIgf-1 or Ikk2 ${ }^{\text {mko }}$ mice (Figure $7, \mathrm{~B}-\mathrm{J}$ ), and both MuRF1 and atrogin-1/MAFbx levels (Figure 7, K and L) were reduced in the double mutants. Therefore, combining IGF-1 supplementation with NF-אB with inhibition further reduces the pathological symptoms of muscle atrophy.

Muscle-specific deletion of IKK enhances regeneration. To determine whether NF-KB muscle-specific inhibition could contribute to muscle regeneration, mouse $\mathrm{TA}$ and $\mathrm{Q}$ muscles were injected with CTX and allowed to recover for 2, 5, or 10 days. Histological analysis of the TA 2 days after injury (Figure 8, A and B) revealed that the CTX injection caused comparable local damage in the different genotypes, indicated by the similar necrotic structure and high infiltration due to initiation of the inflammatory process. However, at day 5 after injury (Figure 8, C and D), although the number of myofibers with centralized nuclei (indicative of muscle regeneration) close to the area of injury was similar (Figure $8 \mathrm{H}$ ), their size was significantly increased, as revealed by the larger CSA of the regenerative fibers (Figure 8, D and G), suggesting that the recovery of the Ikk $2^{\text {mko }}$ muscles was enhanced. By day 10, regeneration in the $I k k 2^{\text {mko }}$ muscles was more profound, demonstrating improved morphology, reduced intracellular space, and larger size of new myofibers (Figure 8, E-F). Even in high dosages of CTX injection, the $I k k 2^{m k o}$ muscles displayed a relatively better morpho- muscle, most of the regenerating fibers contained 1 centralized nucleus 10 days after injury, and very few fibers (Figure 9E) contained more than 1 centralized located nuclei. In contrast, muscles of the Ikkmo/desmin-LacZ injured mice contained many fibers with 2 or 3 centralized nuclei (Figure 9F). The increased multinucleate regenerating fibers of the Ikk2 $2^{\text {moo }}$ desmin-nLacZ mice suggest that IKK2 muscle depletion enhances muscle reconstitution by increasing the number of myonuclei in the damaged myofiber.

Further exploration of the potential regeneration mechanisms enhanced by IKK 2 ablation revealed that Ikk2 $2^{m k o}$ injured muscles accumulated relatively smaller fibrotic invasions compared with the control injured muscles (Figure 8, E and F, and Supplemental Figure 3). Fibrotic tissue is the result of stimulation by cytokines produced by infiltrating cells during inflammation following injury (24). Immunostaining of injured muscles revealed the presence of numerous activated inflammatory cells (as indicated by MHCII staining) in both control and Ikk2 $2^{\text {mo }}$ muscles at day 2 after injury (data not shown) while at later time points, a more

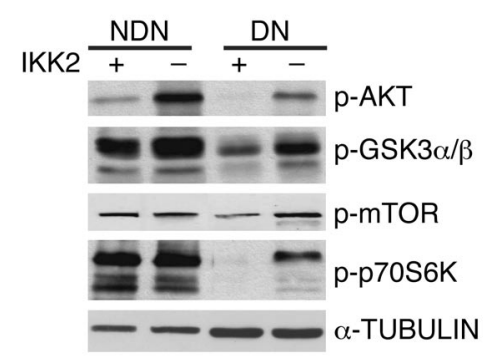

\section{Figure 6}

Immunoblot analysis of protein extracts from control $\left(I k k 2^{t / f}\right)$ and $l k k 2^{m k o}$ denervated and nondenervated muscles stained for anti-p-Akt Ser472/473, anti-p-GSK3 $\alpha / \beta$ Ser21/9, anti-p-mTOR Ser 2448, and anti-p-p70S6K Thr421/Ser424. Anti-tubulin served as a loading control. 
A
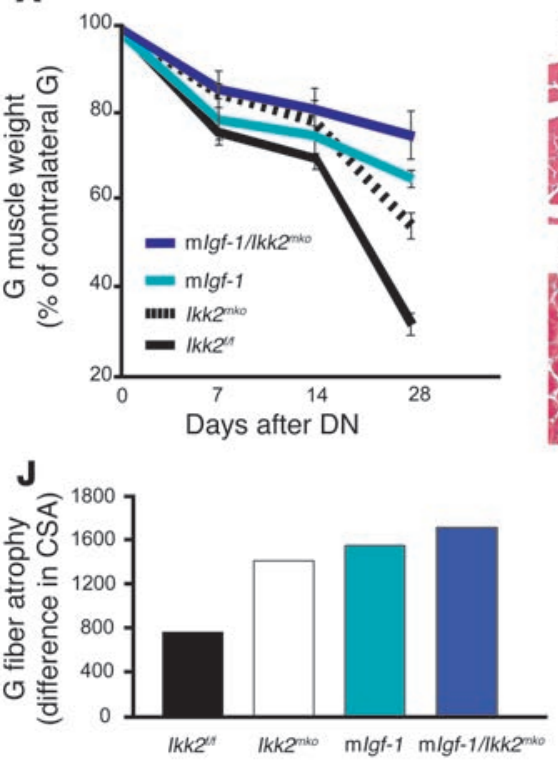

Ikk2
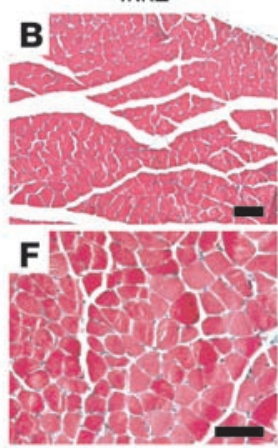

K

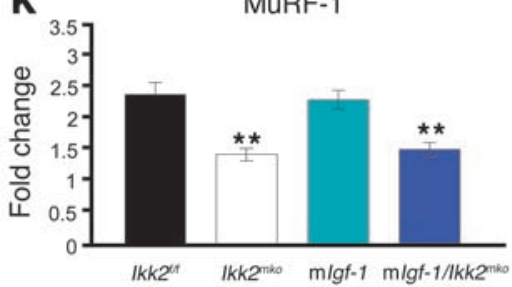

G

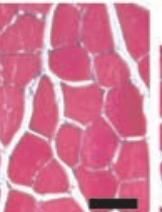

mlgf-1
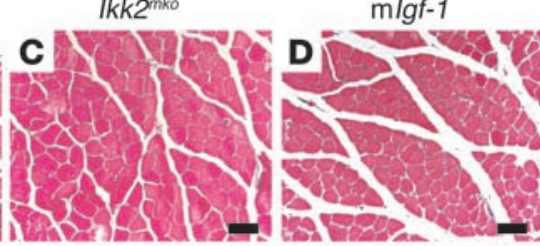

$\mathrm{m} / g f-1 / l k k 2^{m k o}$
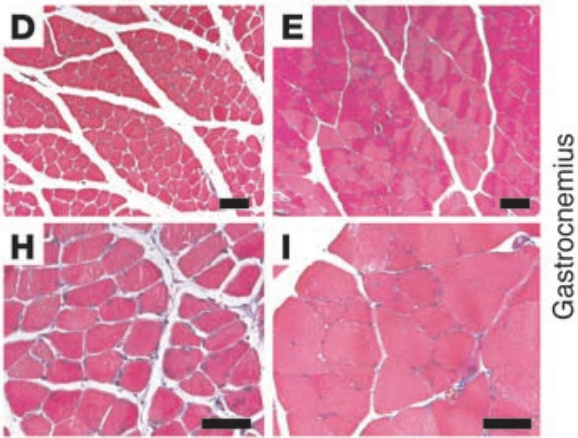

L

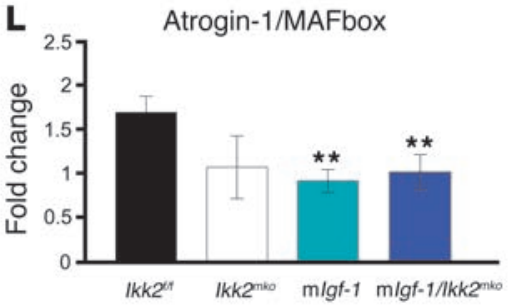

Figure 7

Combined IGF-1 supplementation and IKK2 inhibition enhances protection against muscle atrophy. (A) Comparison of gastrocnemius weights from control (black line), lkk2mko (dashed line), m/gf-1 (light blue line), and m/gf-1/lkk2fff (dark blue line) mice at 7, 14, and 28 days after denervation. Values represent denervated muscle weights after normalization to sham-operated muscle weight ( $n=5$ mice per time point). (B-I) Enhanced maintenance of muscle-fiber size in gastrocnemius muscle from $\mathrm{m} / \mathrm{gf}-1$ and $\mathrm{m} / \mathrm{gf}-1 / \mathrm{lkk} 2^{f / f}$ denervated muscle. Magnification, $\times 100$ (B-E); $\times 200$ (F-I). (J) Sustenance in the gastrocnemius fiber CSA in lkk2 ${ }^{m k o}, \mathrm{~m} / g f-1$, and $\mathrm{m} / g f-1 / l k k 2^{f / f}$ atrophied muscle. (K) MuRF1 and (L) atrogin-1/MAFbx mRNA levels in gastrocnemius muscles of control $(n=4)$, Ikk2mko $(n=4), m / g f-1(n=3)$, and $m / g f-1 / l k k 2^{f / f}(n=4)$ muscles as determined by quantitative real-time RT-PCR. Denervated values were normalized to nondenervated values. ${ }^{* *}$ Mean values differ significantly from those of control $(P<0.002)$.

rapid reduction in activated inflammatory cells in the $I k k 2^{m k o}$ muscles was seen (Figure 10, A-C). These data show how musclespecific IKK2 depletion accelerates regeneration in part by limiting the inflammatory response.

\section{Discussion}

The prolongation of skeletal muscle strength has been the objective of numerous gene- and cell-based therapeutic approaches, which provide useful starting points for treating muscle wasting.
The data presented here establish NF- $\mathrm{BB}$ as an important modulator of normal muscle homeostasis, in that depletion of IKK2 produces muscles with increased strength, greater muscle force, and longer endurance. Since to date a mouse model of muscle cachexia is lacking, currently available animal models of muscle damage and disuse atrophy were employed to investigate the physiological properties of IKK2-deficient muscle under pathological conditions. The reduction in denervation-induced atrophy of $I k k 2^{m k o}$ muscles confirmed the negative impact of NF-KB
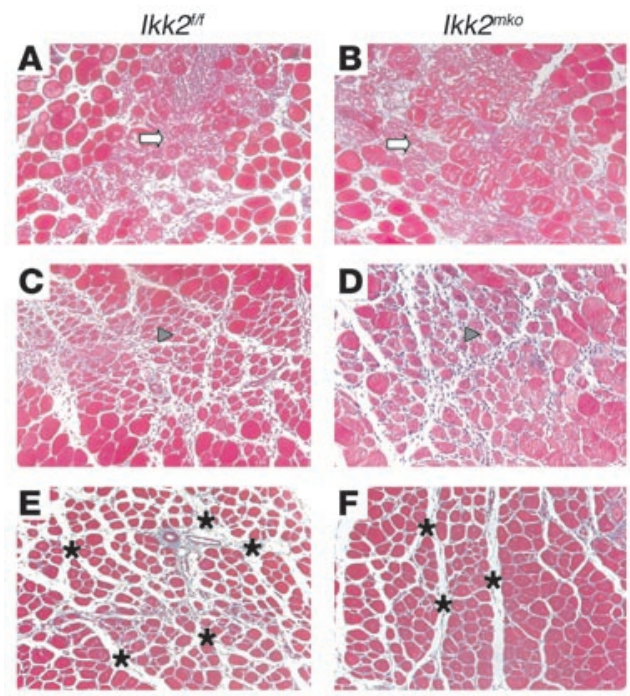

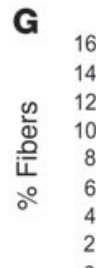

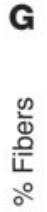

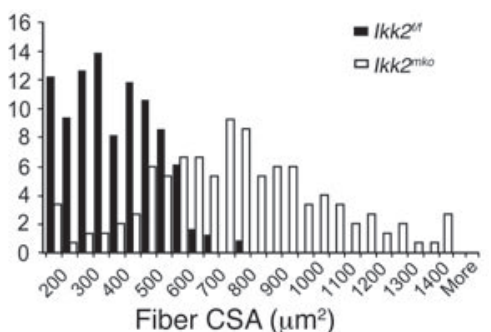

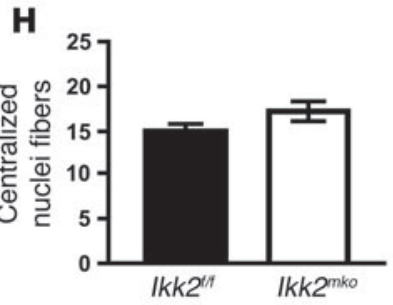

\section{Figure 8}

IKK2 depletion promotes muscle regeneration. (A-F) Trichrome staining of injured TA muscle sections from $I k k 2^{f / f}$ and $l k k 2^{m k o}$ mice. (A and $\mathbf{B}$ ) Extensive necrosis in both genotypes 2 days after CTX injection. Areas with infiltrated cells are marked with white arrows. (C and D) Recovery of the injured muscles in regenerating myofibers containing centralized nuclei (arrowheads) 5 days after injury. (E and F) Improved Ikk2 ${ }^{m k o}$ fiber morphology after 10 days. Intercellular space is marked with asterisks. (G) Increased CSA in the $l k k 2^{m k o}$ regenerated fibers. (H) Regenerating myocytes containing centralized nuclei close to injured area in the $l k k 2^{m k o}$ injured muscles $(n=5)$. 


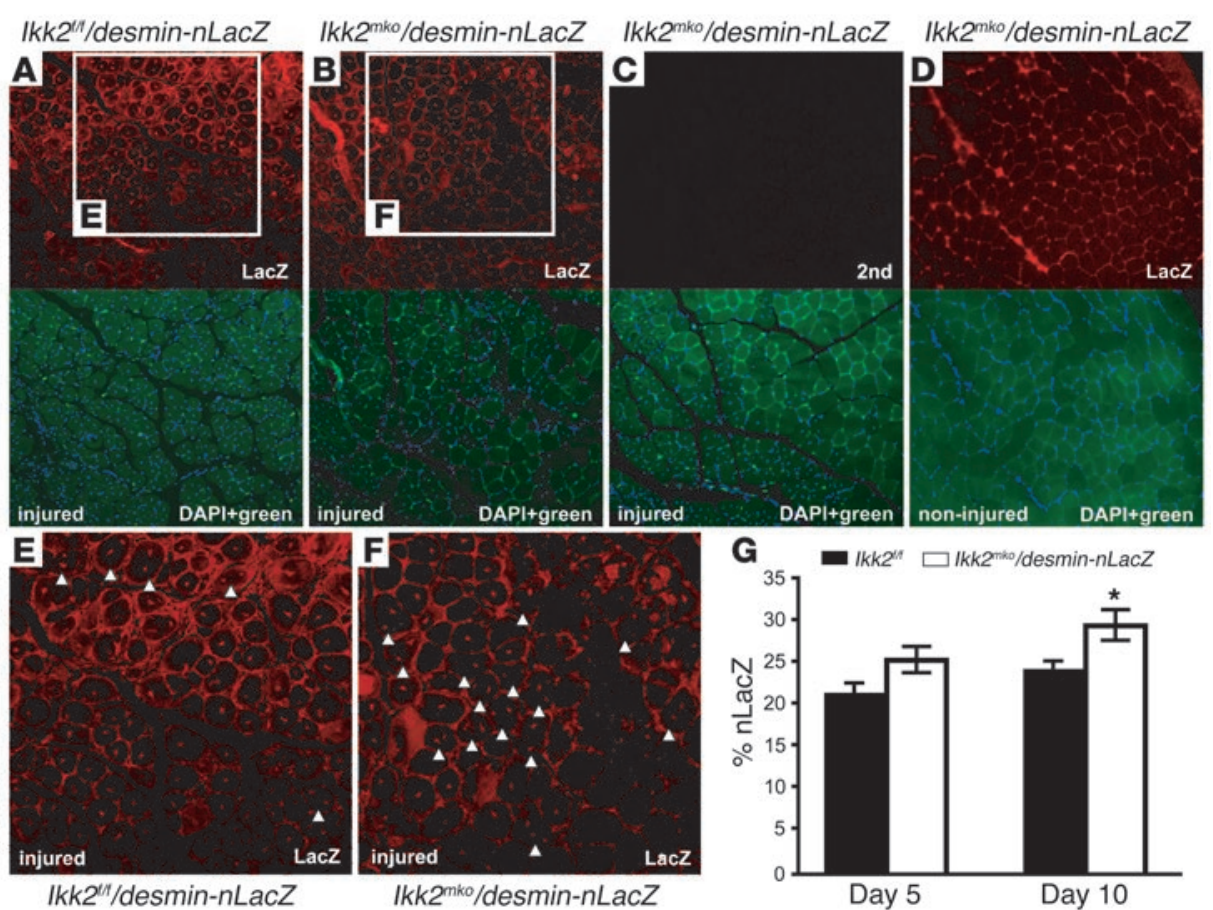

Figure 9

IKK2 muscle deletion increases multinucleate regenerating fibers. (A and B) Anti-LacZ immunostaining (red) of regenerating muscles (day 10 ) from control and $l k k 2^{m k o}$ mice. (C) Negative control using only the secondary antibody (2nd) (no staining in red) in the lkk2mko regenerating muscle. (D) Anti-LacZ immunostaining (red) of noninjured $l k k 2^{m k o}$ muscle. (A-D, lower panels) DAPI-counterstained (blue staining) sections merged with green fluorescence (no staining). (E-F) Enlargement of regenerating areas. Note the increased desmin-nLacZ-positive cells in the regenerating area of $I k 2^{m k o}$ sections. Examples of fibers with double- or triple-LacZ-positive nuclei are indicated with arrowheads. (G) Graph showing nLacZ-positive nuclei counted from 3 independent injured mice, presented as a percentage of total DAPI nuclei. *Mean value differs significantly from that of control $(P<0.05)$. Magnification, $\times 100$ (A-D); $\times 200$ (E and F).

signaling on muscle wasting (21). Moreover, the improved regenerative responses of $I k k 2^{\text {mko }}$ muscles after local muscle injury revealed for what we believe is the first time a link between muscle repair and NF- $\kappa \mathrm{B}$ inhibition.

The involvement of IKK/NF-кB in muscle physiology has previously been demonstrated in in vitro cell culture models (17-20, 34-37) or in use of transgenic muscle with specific overexpression of IкB $\alpha$ super-repressor (21). Numerous reports $(35,37-40)$ have associated TNF- $\alpha$ and NF- $\kappa$ B with muscle wasting diseases and muscle atrophy (21). Denervation-induced atrophy generally results in rounded atrophied fibers with smaller CSAs. However, typical angular atrophic fibers are also a result of shrinkage. The muscle attempts to compensate for tissue loss by switching to a fast-twitch phenotype (4, 28, 41). In the case of the Ikk2 $2^{\text {mko }}$ mouse model presented here, muscle atrophy even 1 month after denervation was minimal, and angular atrophied fibers were rarely observed, with a marked absence of fiber switching during the progression of the disease. Consequently, muscle force in the Ikk $2^{\text {mko }}$ denervated mice remained high, most likely due to a synergistic effect of maintaining both muscle mass and fiber integrity. Thus, NF-кB blockade attenuates the major pathological features of denervation-induced atrophy (fiber-size reduction, fibertype switching, and muscle-force alteration).

Maintenance of muscle mass is controlled as a balance between protein synthesis and protein degradation pathways (8). In a patho- physiological setting such as denervation, signals that promote protein synthesis, such as those involving mTOR and GSK3 $\alpha / \beta(42,43)$, are normally suppressed, and protein degradation pathways involving ubiquitin ligases are activated. Protection against these signaling imbalances in $I k k 2^{\text {mko }}$ denervated muscle prevents muscle atrophy. Of particular interest is the increased activation of the survival kinase Akt since previous reports on other cell types show that Akt activates NF-кB (44). The crosstalk between Akt and NF-KB pathways in muscle has important implications for treatment of muscle wasting. Further complexity is revealed in the regulation of ubiquitin ligases, where NF-кB inhibition suppresses the induction of the MuRF1 while the expression of atrogin-1/MAFbx is probably NF- $\mathrm{KB}$ independent. Moreover, NF-KB inhibition combined with IGF-1, which represses atrogin-1/MAFbx, further promotes muscle sparing. A working model of NF- $\mathrm{KB}$ inhibition is proposed, in which IKK2 depletion restabilizes a new ratio of protein synthesis/degradation in response to the changes caused by muscle atrophy without affecting insulin and glucose homeostasis (45).

Using the well-established CTX model of skeletal muscle injury, we demonstrated that NF- $\kappa \mathrm{B}$ inhibition reduced inflammation and enhanced myoblast contribution after local muscle damage, providing definitive evidence that NF- $\mathrm{KB}$ inhibition accelerates the muscle regeneration process. The faster healing rates of $I k k 2^{\text {mko }}$ injured muscles were accompanied by decreased fibrosis, demonstrating that NF- $\mathrm{KB}$ inhibition permits rapid restoration of tissue architecture. The increased size of the regenerating myofibers in Ikk2 $2^{m k o}$ injured muscles also suggests that growth and/or differentiation is affected. These findings are consistent with cell culture experiments in which induction of NF- $\mathrm{\kappa B}$ inhibits myogenic differentiation and blocks the muscle transcription factor MyoD (19, 20, 34).

During the muscle regeneration process, preexisting satellite cells enter the necrotic segments after myofiber disruption, where they proliferate, differentiate, and form new fibers. Satellite cells are also mobilized to migrate from the surrounding tissue into the regenerating areas, participating in the reconstitution of skeletal muscle fibers (46). In our studies, the majority of $I k k 2^{\text {mko }}$ injured fibers were replaced mainly by new fibers and to a lesser extent by connective tissue, as defined by histological analysis and an increase in the number of desmin-nLacZ-positive cells. Since NF-кB has been previously shown to regulate cell cycle genes (17), satellite cell proliferation might also contribute to the increase in LacZ-positive cells in the Ikk2 ${ }^{\text {mko }}$ regenerating muscles. It is likely 

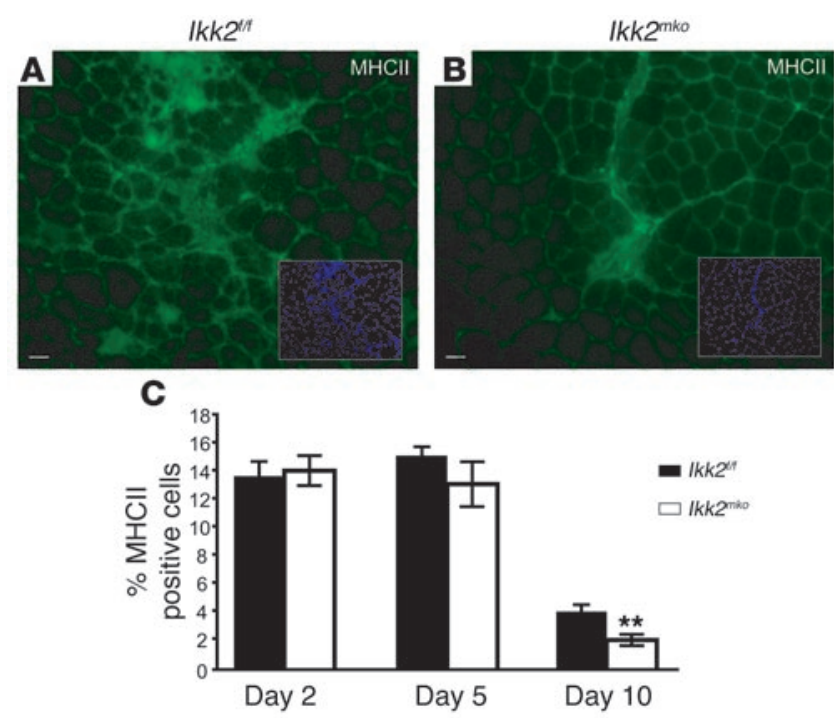

Figure 10

Muscle-specific IKK2 depletion limits the inflammatory response. (A and $\mathbf{B}$ ) Anti-MHCII antibody was used to identify infiltrating cells from 3 independent injured mice (10 days after injury). (C) The graph represents the percentage of anti-MHCll cells at all time points as a percentage of the total DAPI nuclei. Note that at day 10, the number of infiltrating cells was significantly decreased in the $I k k^{m k o}$ injured muscles. ${ }^{* *}$ Mean value differs significantly from that of control $(P<0.002)$.

that additional factors, such as enhanced fusion, may contribute to the regenerative process in the absence of NF- $\kappa \mathrm{B}$ activity. In fact, injury of $\mathrm{Ikk}^{\mathrm{mko}}$ muscle produced no increase in the number of regenerating fibers (as marked by centralized nuclei) but rather an increased number of centralized nuclei per fiber. This suggests that fusion and hypertrophic growth have been enhanced through disruption of NF-KB signaling.

The restriction of IKK2 gene inactivation to the skeletal muscle excludes the direct involvement of other cell types in the muscle environment, such as fibroblasts and vascular tissue, explaining the discrepancies seen in other studies (47-50), in which total knockouts or pharmacological inhibitors against molecules of the NF- $\kappa B$ signaling pathway were used to study muscle repair. Our observations, along with the documented upregulation of NF-кB in Duchenne muscular dystrophy patients (51) and in the corresponding mdx mouse model (52), suggest a negative role of NF-KB in the development of this devastating disease. Indeed, the current study provides evidence that NF- $\mathrm{BB}$ contributes to muscle damage and explains the amelioration of muscle healing by IKK selective inhibition in other studies (53). The broad benefits of NF-KB blockade in augmenting muscle performance raise new and exciting possibilities for therapeutic approaches in the treatment of muscular disorders and for the development of strategies to attenuate human muscle wasting.

\section{Methods}

Animals. Mice expressing Cre recombinase under control of the muscle creatine kinase (MCK) promoter (27) were crossed with Ikk2f/f mice (26, $45)$. Animals were housed in a clean, temperature-controlled $\left(22^{\circ} \mathrm{C}\right)$ mouse facility on a 12-hour light/dark cycle, and standard diet was provided. Genotyping was performed by PCR using genomic DNA from tail digests as previously described $(26,54)$. For denervation and regeneration experiments, animals were anesthetized using $2.5 \%$ Avertin. All mouse procedures were approved by the European Molecular Biology Laboratory Monterotondo Ethical Committee (Monterotondo, Italy) and were in accordance with national and European regulations.

EMSA. Nuclear extracts $(4 \mu \mathrm{g})$ of the $\mathrm{Q}$ and gastrocnemius were incubated for 30 minutes at room temperature with $2 \mu \mathrm{g}$ poly(dI-dC) (Pharmacia) and $0.5 \mathrm{ng}$ of ${ }^{32} \mathrm{P}$-labeled $\kappa \mathrm{B}$ probe (prepared from $\mathrm{H} 2-\kappa \mathrm{B}$-site primer: 5'-CGGGCTGGGGATTCCCCATCTCGGTAC-3' and 5'-GTACCGAGATGGGGAATCCCCAGCCCG-3'). Samples were fractionated on a $5 \%$ PAGE for 3 hours and visualized by autoradiography. For super shift experiments, antibodies against p50 and p65 (Santa Cruz Biotechnology Inc.) were used.

IKK assay. Protein extract $(300 \mu \mathrm{g})$ was immunoprecipitated with $2 \mu \mathrm{l}$ anti-NEMO antibody (Santa Cruz Biotechnology Inc.), and the kinase assay was performed as described previously (55), using a glutathione S-transferase-IкB (aa residues 1-54) substrate.

Histology. For cryosection, different muscles of the 4-month-old animals were embedded in gum tragacanth (Sigma-Aldrich), frozen in isopentane, chilled in liquid nitrogen, and stored at $-80^{\circ} \mathrm{C}$. Transverse cross sections of 5 - and $7-\mu \mathrm{m}$ thick were collected from the middle of each muscle using a cryostat (Cryostat Leica CM3050 S; Leica Microsystems). NADH staining was performed as previously described (56). For paraffin sections (Leica RM 2135 microtome), muscles were fixed in 4\% PFA, dehydrated in a series of ethanol dilutions, passed through xylene and xylene/ paraffin, and finally embedded in paraffin. Paraffin sections were cut at $7 \mu \mathrm{m}$ and subjected to either H\&E (Sigma-Aldrich) or trichrome staining (Sigma-Aldrich). Slides were mounted using Aqua Mount medium (Lerner Laboratories; Fisher Scientific), and photos were collected with a Leica DC 500 camera (Leica Microsystems). The CSA was analyzed using MetaMorph software (version 6.2r5; Universal Imaging Corp.).

Immunohistochemistry. Cryosections were fixed for 10 minutes in 4\% PFA on ice, washed once with PBT, blocked with PBT, $0.3 \%$ Triton X-100, and $5 \%$ normal goat serum for 1 hour at room temperature and incubated with primary antibody overnight at $4{ }^{\circ} \mathrm{C}$ in blocking solution. Paraffin sections were deparaffinized in xylene and dehydrated in a series of ethanol dilutions. The antigen retrieval was performed with $\mathrm{Na}$ citrate $(10 \mathrm{mM}$, $\mathrm{pH}$ 9.0) for 30 minutes at $80^{\circ} \mathrm{C}$. After washing in PBT, the sections were blocked with $5 \%$ horse serum and incubated with the primary antibody (in blocking solution) overnight at $4{ }^{\circ} \mathrm{C}$. The sections were washed briefly with PBT before incubation with secondary antibody for 1 hour at room temperature and then washed 3 times for 40 minutes with PBT. The slides were mounted using medium for fluorescence with DAPI (Vector Laboratories), and pictures were taken using a Leica DC500 microscope. The primary antibodies that were used are as follows: anti- $\beta$ galactosidase $(1: 1,000$, rabbit; MP Biomedicals Inc.) and anti-MHCII (1:50, mouse; Biozol). The secondary antibodies were anti-mouse Cy3 conjugate (1:100; Jackson ImmunoResearch Laboratories Inc.) and anti-rabbit Cy2 conjugate (1:100; Jackson ImmunoResearch Laboratories Inc.).

Muscle denervation. The right hind limb of 4-month-old anesthetized male mice was denervated via sciatic nerve section ( $n=5 /$ group). The contralateral hind limb was used as a control. Mice were provided food and water ad libitum and were sacrificed after 7,14 , or 28 days; individual muscles were weighed. Some of the muscles were quick frozen to the temperature of liquid nitrogen for RNA and protein isolation and others used for histological analysis.

Muscle regeneration. Two muscles, TA and Q, from 3-month-old Cre-negative (control) and Cre-positive (Ikk2 $\left.2^{m k o}\right)$ mice were injected with $25 \mu \mathrm{l}$ and $40 \mu \mathrm{l}$, respectively, of $10 \mu \mathrm{M}$ CTX (Latoxan, diluted in PBS). The muscles were collected 2, 5, and 10 days following the injection of CTX. At least 6 mice per time point were analyzed. For the satellite cell mobilization experiment, the desmin/nuclear localization signal-LacZ transgene (33) was crossed to the Ikk2 $2^{m k o}$ animals. 
Quantitative real-time RT-PCR. Total RNA was extracted from muscles using TRIzol (Invitrogen), and cDNA was synthesized using Ready-ToGo T-Primed First-Strand Kit (Amersham Biosciences). Primers were as follows: 5'-CAGATTCTCCTTACTGTATACCTCCTTGT-3' and 5'GGCGGACGGCTGAA-3' for atrogin-1; 5'-AGGACAACCTCGTGCCTACAAG-3' and 5'-ACAACCTGTGCCGCAAGTG-3' for MuRF1. PCR amplifications were quantified using the DyNAmo SYBR Green qPCR Kit (FINNZYMES). Results were normalized against ubiquitin, $\beta$-tubulin, and GAPDH expression.

Exercise tolerance tests. Mice were subjected to an exhaustion treadmill test. Each mouse was placed on the belt of a 6-lane motorized treadmill (Exer 3/6 Treadmill; Columbus Instruments) supplied with shocker plates. The treadmill was run at an inclination of $0^{\circ}$ at $5 \mathrm{~m} / \mathrm{min}$ for 5 minutes, after which the speed was increased by $1 \mathrm{~m} / \mathrm{min}$ every minute. The test was stopped when the mouse remained on the shocker plate for more than 20 seconds without attempting to reengage the treadmill, and the time to exhaustion was determined.

Intact muscle mechanical measurement. Animals were killed by cervical dislocation. EDLs were dissected as entire muscles and were mounted in the organ bath with 1 tendon tied to a force transducer and the other tendon tied to a length adapter. Muscles were bathed in a $95 \% \mathrm{O}_{2} / 5 \% \mathrm{CO}_{2}$ perfused Krebs solution at $22^{\circ} \mathrm{C}$ and stimulated directly by an electric field generated by a stimulator (S48; Grass Telefactor), split (Stimu-Splitted II; Med-Lab), and controlled by an attenuator (4-Channel Attenuator; Med-Lab). Muscles were adjusted to optimum length (Lo) before all force measurements. For comparative purposes all force measurements were expressed per total muscle unit CSA calculated by dividing the muscle mass by the product of length and the mammalian skeletal muscle density $\left(1.06 \mathrm{mg} / \mathrm{mm}^{3}\right)$.

Phosphorylated protein screen. Protein samples from the gastrocnemius of $I k k 2^{m k o}$ and $I k k 2^{f / f}$ controls were probed for the quantitative expression of 46 phosphorylated proteins (p-proteins) using validated commercial antibodies in the Kinetworks KPSS 4.1 immunoblotting analysis provided by Kinexus Bioinformatics Corp. Samples were resolved on $12.5 \%$ SDS-PAGE gels followed by electrophoretic transfer to a thin support membrane. The membranes were then probed with antibody mixes that had been thoroughly validated for their potency and specificity in human, mouse, and rat. Bound antibodies were detected using the ECL Detection System. Three mice were used for each genotype and the results expressed as the average fold induction over the value of the nondenervated gastrocnemius for each phosphorylated site.

Immunoprecipitation. Protein extracts from muscles were immunoprecipitated with polyclonal antibodies against NEMO (Santa Cruz Biotechnology Inc.), incubated with protein $\mathrm{A} / \mathrm{G}$ beads (Oncogene Science), washed, and resuspended in Laemmli sample buffer.

Immunoblotting. Protein extraction was performed in RIPA buffer: $1 \%(\mathrm{w} / \mathrm{w})$ Nonidet P40; $1 \%(\mathrm{w} / \mathrm{w})$ sodium deoxylate; $0.1 \%(\mathrm{w} / \mathrm{v})$ SDS;
$150 \mathrm{mM} \mathrm{NaCl} ; 50$ mM HEPES, pH 7.0; 2 mM EDTA, pH 8.0; $100 \mathrm{mM}$ $\mathrm{NaF} ; 10 \%$ glycerol; $1.5 \mathrm{mM} \mathrm{MgCl} 2 ; 100 \mathrm{mM}$ PMSF in ethanol; $200 \mathrm{mM}$ sodium orthovanadate; and $1 \mu \mathrm{g} / \mathrm{ml}$ aprotin. Protein determination was performed with the Bradford method (Bio-Rad), and equal amounts of protein from each muscle lysate were separated in SDS polyacrylamide minigel (Bio-Rad) and transferred onto a hybond ECL nitrocellulose membrane (Amersham Biosciences). Filters were blocked with 5\% milk, blotted with specific antibodies and incubated overnight at $4{ }^{\circ} \mathrm{C}$, washed 3 times for 30 minutes with washing buffer (TBS and $0.1 \%$ Tween-20), and blotted with secondary antibodies (peroxidase-conjugated) (Amersham Biosciences in 1:7,000 dilution) with 5\% milk for 1 hour at room temperature. The filter was incubated for 1 minute using ECL kit (Amersham Biosciences) before exposure. Primary antibodies were as follows: antiIKK1 and anti-IKK2 (1:250; IMGENEX), anti-NEMO (1:500; Pasparakis lab), anti-p-Akt Ser472/473 (1:1,000, BD Biosciences - Pharmingen), anti-p-GSK3 $\alpha / \beta$ Ser21/9 (1:1,000; Cell Signaling Technology), antip-mTOR Ser 2448 (1:1,000; Cell Signaling Technology), anti-p-p70S6K Thr421/Ser424 (1:1,000; Cell Signaling Technology), and anti-tubulin (1:2,000; Sigma-Aldrich).

Statistics. Data were analyzed by ANOVA followed by post-hoc comparisons using the Fisher's test in cases of statistical significance. $P$ values less than 0.05 were considered significant. All analyses were carried out using StatView 5.0 statistical software (StatSoft).

\section{Acknowledgments}

We thank E. Slonimsky for technical assistance; E. Perlas for advice and protocols concerning the histological analysis; and J. Gonzales, P. Giallonardo, L. Tatangel, and especially M. D'ercole for animal care. We are grateful to T. Tsetsenis, A. Musaro, and members of the Rosenthal and Pasparakis labs for critical comments and helpful discussions. We thank J. Bruning and R. Kahn for providing the MCKCre mice. This work was supported by grants to N. Rosenthal from the Muscular Dystrophy Association, the Leducq Foundation for Transatlantic Research, and the MYORES European Muscle Development Network as well as EU grant LSHG-CT2005-005203 to M. Pasparakis.

Received for publication April 3, 2006, and accepted in revised form July 25, 2006.

Address correspondence to: Foteini Mourkioti, EMBL Mouse Biology Unit, Campus “A. Buzzati-Traverso,” via Ramarini 32, 00016, Monterotondo-Scalo (RM), Italy. Phone: 39-06-90091-214; Fax: 39-06-90091-272; E-mail: fmourkioti@embl-monterotondo.it.

\section{Pasparakis and N. Rosenthal are co-senior authors.}

1. Nowak, K.J., and Davies, K.E. 2004. Duchenne muscular dystrophy and dystrophin: pathogenesis and opportunities for treatment. EMBO Rep. 5:872-876.

2. Strassburg, S., Springer, J., and Anker, S.D. 2005. Muscle wasting in cardiac cachexia. Int. J. Biochem. Cell Biol. 37:1938-1947.

3. Filippatos, G.S., Anker, S.D., and Kremastinos, D.T 2005. Pathophysiology of peripheral muscle wasting in cardiac cachexia. Curr. Opin. Clin. Nutr. Metab. Care. 8:249-254.

4. Tisdale, M.J. 2002. Cachexia in cancer patients. Nat. Rev. Cancer. 2:862-871.

5. Dalakas, M.C. 2004. Inflammatory disorders of muscle: progress in polymyositis, dermatomyositis and inclusion body myositis. Curr. Opin. Neurol. 17:561-567.
6. Mitch, W.E., and Goldberg, A.L. 1996. Mechanisms of muscle wasting. The role of the ubiquitin-proteasome pathway. N. Engl. J. Med. 335:1897-1905.

7. Gomes, M.D., Lecker, S.H., Jagoe, R.T., Navon, A., and Goldberg, A.L. 2001. Atrogin-1, a muscle-specific F-box protein highly expressed during muscle atrophy. Proc. Natl. Acad. Sci. U. S. A. 98:14440-14445.

8. Bodine, S.C., et al. 2001. Identification of ubiquitin ligases required for skeletal muscle atrophy. Science. 294:1704-1708.

9. Price, S.R., et al. 1998. Mechanisms contributing to muscle-wasting in acute uremia: activation of amino acid catabolism. J. Am. Soc. Nephrol. 9:439-443.

10. Price, S.R., et al. 1996. Muscle wasting in insulinopenic rats results from activation of the ATP-dependent, ubiquitin-proteasome proteolytic pathway by a mechanism including gene transcription. J. Clin.
Invest. 98:1703-1708.

11. Tiao, G., et al. 1994. Sepsis stimulates nonlysosomal, energy-dependent proteolysis and increases ubiquitin mRNA levels in rat skeletal muscle. J. Clin. Invest. 94:2255-2264.

12. Degens, H., and Alway, S.E. 2006. Control of muscle size during disuse, disease, and aging. Int. J. Sports Med. 27:94-99.

13. Karin, M., Yamamoto, Y., and Wang, Q.M. 2004. The IKK NF-kappa B system: a treasure trove for drug development. Nat. Rev. Drug Discov. 3:17-26.

14. Zandi, E., Rothwarf, D.M., Delhase, M., Hayakawa, M., and Karin, M. 1997. The IkappaB kinase complex (IKK) contains two kinase subunits, IKKalpha and IKKbeta, necessary for IkappaB phosphorylation and NF-kappaB activation. Cell. 91:243-252.

15. Yamaoka, S., et al. 1998. Complementation clon- 
ing of NEMO, a component of the IkappaB kinase complex essential for NF-kappaB activation. Cell. 93:1231-1240

16. Ghosh, S., and Karin, M. 2002. Missing pieces in the NF-kappaB puzzle. Cell. 109(Suppl.):S81-S96.

17. Guttridge, D.C., Albanese, C., Reuther, J.Y., Pestell, R.G., and Baldwin, A.S., Jr. 1999. NF-kappaB controls cell growth and differentiation through transcriptional regulation of cyclin D1. Mol. Cell. Biol. 19:5785-5799.

18. Li, Y.P. 2003. TNF-alpha is a mitogen in skeletal muscle. Am. J. Physiol. Cell Physiol. 285:C370-C376.

19. Guttridge, D.C., Mayo, M.W., Madrid, L.V., Wang, C.Y., and Baldwin, A.S., Jr. 2000. NF-kappaB-induced loss of MyoD messenger RNA: possible role in muscle decay and cachexia. Science. 289:2363-2366.

20. Langen, R.C., et al. 2004. Tumor necrosis factor-alpha inhibits myogenic differentiation through MyoD protein destabilization. FASEB J. 18:227-237.

21. Cai, D., et al. 2004. IKKbeta/NF-kappaB activation causes severe muscle wasting in mice. Cell. 119:285-298.

22. Tanaka, M., et al. 1999. Embryonic lethality, liver degeneration, and impaired NF-kappa B activation in IKK-beta-deficient mice. Immunity. 10:421-429.

23. Li, Q., Van Antwerp, D., Mercurio, F., Lee, K.F., and Verma, I.M. 1999. Severe liver degeneration in mice lacking the IkappaB kinase 2 gene. Science. 284:321-325.

24. Mourkioti, F., and Rosenthal, N. 2005. IGF-1, inflammation and stem cells: interactions during muscle regeneration. Trends Immunol. 26:535-542.

25. Musaro, A., et al. 2001. Localized Igf-1 transgene expression sustains hypertrophy and regeneration in senescent skeletal muscle. Nat. Genet. 27:195-200.

26. Pasparakis, M., et al. 2002. TNF-mediated inflammatory skin disease in mice with epidermis-specific deletion of IKK2. Nature. 417:861-866

27. Bruning, J.C., et al. 1998. A muscle-specific insulin receptor knockout exhibits features of the metabolic syndrome of NIDDM without altering glucose tolerance. Mol. Cell. 2:559-569.

28. D'Antona, G., et al. 2003. The effect of ageing and immobilization on structure and function of human skeletal muscle fibres. J. Physiol. 552:499-511.

29. Ijkema-Paassen, J., Meek, M.F., and Gramsbergen, A. 2005. Long-term reinnervation effects after sciatic nerve lesions in adult rats. Ann. Anat. 187:113-120.

30. Glass, D.J. 2003. Signalling pathways that mediate skeletal muscle hypertrophy and atrophy. Nat. Cell Biol. 5:87-90.

31. Stitt, T.N., et al. 2004. The IGF-1/PI3K/Akt pathway prevents expression of muscle atrophy-induced ubiquitin ligases by inhibiting FOXO transcription factors. Mol. Cell. 14:395-403.

32. Sandri, M., et al. 2004. Foxo transcription factors induce the atrophy-related ubiquitin ligase atrogin-1 and cause skeletal muscle atrophy. Cell. 117:399-412.

33. Lescaudron, L., Creuzet, S.E., Li, Z., Paulin, D., and Fontaine-Perus, J. 1997. Desmin-lacZ transgene expression and regeneration within skeletal muscle transplants. J. Muscle Res. Cell Motil. 18:631-641.

34. Langen, R.C., Schols, A.M., Kelders, M.C., Wouters, E.F., and Janssen-Heininger, Y.M. 2001. Inflammatory cytokines inhibit myogenic differentiation through activation of nuclear factor-kappaB. FASEB J. 15:1169-1180.

35. Coletti, D., Yang, E., Marazzi, G., and Sassoon, D. 2002. TNFalpha inhibits skeletal myogenesis through a PW1-dependent pathway by recruitment of caspase pathways. EMBO J. 21:631-642.

36. Ladner, K.J., Caligiuri, M.A., and Guttridge, D.C. 2003. Tumor necrosis factor-regulated biphasic activation of NF-kappa B is required for cytokineinduced loss of skeletal muscle gene products. J. Biol. Chem. 278:2294-2303.

37. Di Marco, S., et al. 2005. NF-(kappa)B-mediated MyoD decay during muscle wasting requires nitric oxide synthase mRNA stabilization, HuR protein, and nitric oxide release. Mol. Cell. Biol. 25:6533-6545.

38. Li, Y.P., and Reid, M.B. 2000. NF-kappaB mediates the protein loss induced by TNF-alpha in differentiated skeletal muscle myotubes. Am. J. Physiol. Regul. Integr. Comp. Physiol. 279:R1165-R1170.

39. Hunter, R.B., et al. 2002. Activation of an alternative NF-kappaB pathway in skeletal muscle during disuse atrophy. FASEB J. 16:529-538.

40. Li, Y.P., et al. 2003. TNF-alpha increases ubiquitinconjugating activity in skeletal muscle by up-regulating UbcH2/E220k. FASEB J. 17:1048-1057.

41. Carpenter, S., and Karpati, G. 2001. Pathology of skeletal muscle. Oxford University Press. New York, New York, USA. 662 pP.

42. Bodine, S.C., et al. 2001. Akt/mTOR pathway is a crucial regulator of skeletal muscle hypertrophy and can prevent muscle atrophy in vivo. Nat. Cell Biol. 3:1014-1019.

43. Rommel, C., et al. 2001. Mediation of IGF-1- induced skeletal myotube hypertrophy by $\mathrm{PI}(3) \mathrm{K} /$ Akt/mTOR and PI(3)K/Akt/GSK3 pathways. Nat. Cell Biol. 3:1009-1013.

44. Romashkova, J.A., and Makarov, S.S. 1999. NFkappaB is a target of AKT in anti-apoptotic PDGF signalling. Nature. 401:86-90.

45. Rohl, M., et al. 2004. Conditional disruption of IКB kinase 2 fails to prevent obesity-induced insulin resistance. J. Clin. Invest. 113:474-481. doi:10.1172/ JCI200418712.

46. Schultz, E., Jaryszak, D.L., and Valliere, C.R. 1985. Response of satellite cells to focal skeletal muscle injury. Muscle Nerve. 8:217-222.

47. Chen, S.E., et al. 2005. Role of TNF-alpha signaling in regeneration of cardiotoxin-injured muscle. Am. J. Physiol. Cell Physiol. 289:C1179-C1187.

48. Collins, R.A., and Grounds, M.D. 2001. The role of tumor necrosis factor-alpha (TNF-alpha) in skeletal muscle regeneration. Studies in TNF-alpha(-/-) and TNF-alpha(-/-)/LT-alpha(-/-) mice. J. Histochem. Cytochem. 49:989-1001.

49. Grounds, M.D., and Torrisi, J. 2004. Anti-TNFalpha (Remicade) therapy protects dystrophic skeletal muscle from necrosis. FASEB J. 18:676-682.

50. Thaloor, D., Miller, K.J., Gephart, J., Mitchell, P.O., and Pavlath, G.K. 1999. Systemic administration of the NF-kappaB inhibitor curcumin stimulates muscle regeneration after traumatic injury. Am. J. Physiol. 277:C320-C329.

51. Monici, M.C., Aguennouz, M., Mazzeo, A., Messina, C., and Vita, G. 2003. Activation of nuclear factorkappaB in inflammatory myopathies and Duchenne muscular dystrophy. Neurology. 60:993-997.

52. Kumar, A., and Boriek, A.M. 2003. Mechanical stress activates the nuclear factor-kappaB pathway in skeletal muscle fibers: a possible role in Duchenne muscular dystrophy. FASEB J. 17:386-396.

53. Herrmann, O., et al. 2005. IKK mediates ischemiainduced neuronal death. Nat. Med. 11:1322-1329.

54. Schmidt-Supprian, M., et al. 2000. NEMO/IKK gamma-deficient mice model incontinentia pigmenti. Mol. Cell. 5:981-992.

55. Luedde, T., et al. 2005. Deletion of IKK2 in hepatocytes does not sensitize these cells to TNFinduced apoptosis but protects from ischemia/ reperfusion injury. J. Clin. Invest. 115:849-859. doi:10.1172/JCI200523493.

56. Keilhoff, G., and Fansa, H. 2005. Successful intramuscular neurotization is dependent on the denervation period. A histomorphological study of the gracilis muscle in rats. Muscle Nerve. 31:221-228. 